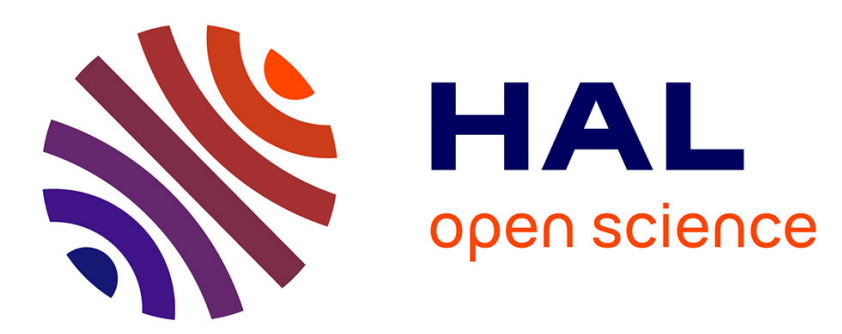

\title{
Impact of Fluorocarbon Gaseous Environments on the Permeability of Foam Films to Air
}

Céline Hadji, Benjamin Dollet, Hugues Bodiguel, Wiebke Drenckhan, Benoit Coasne, E. Lorenceau

\section{- To cite this version:}

Céline Hadji, Benjamin Dollet, Hugues Bodiguel, Wiebke Drenckhan, Benoit Coasne, et al.. Impact of Fluorocarbon Gaseous Environments on the Permeability of Foam Films to Air. Langmuir, 2020, 10.1021/acs.langmuir.0c02158 . hal-02997264

\section{HAL Id: hal-02997264 \\ https://hal.science/hal-02997264}

Submitted on 10 Nov 2020

HAL is a multi-disciplinary open access archive for the deposit and dissemination of scientific research documents, whether they are published or not. The documents may come from teaching and research institutions in France or abroad, or from public or private research centers.
L'archive ouverte pluridisciplinaire $\mathbf{H A L}$, est destinée au dépôt et à la diffusion de documents scientifiques de niveau recherche, publiés ou non, émanant des établissements d'enseignement et de recherche français ou étrangers, des laboratoires publics ou privés. 


\title{
Impact of fluorocarbon gaseous environments on the permeability of soap films to air
}

\author{
Céline Hadji, ${ }^{* \dagger}$ Benjamin Dollet, ${ }^{\dagger}$ Hugues Bodiguel, ${ }^{\ddagger}$ Wiebke Drenckhan, \\ Benoît Coasne, ${ }^{\dagger}$ and Elise Lorenceau, ${ }^{*}$ \\ $\dagger$ Univ. Grenoble Alpes, CNRS, LIPhy, F-38000 Grenoble, France \\ $\ddagger$ Univ. Grenoble Alpes, Grenoble-INP, CNRS, LRP UMR5520, France \\ IUniv. Strasbourg, CNRS, Institut Charles Sadron, UPR22, F-67000 Strasbourg, France \\ E-mail: celine.hadji@gmail.com; elise.lorenceau@univ-grenoble-alpes.fr
}

\section{Abstract}

A mobile soap film, stabilized with tetradecyltrimethylammonium bromide (TTAB) or sodium dodecylsulfate (SDS) surfactants and deposited in the middle of a cylindrical tube separates the tube into two distinct gaseous compartments. The first compartment is filled with air while the second one contains a mixture of air and perfluorohexane $\left(\mathrm{C}_{6} \mathrm{~F}_{14}\right)$, which is a fluorinated gas barely soluble in water. This soap film thus acts as a liquid semi-permeable membrane for gases equivalent to solid semipermeable membranes conventionally used in fluid separation processes. To infer the rate of air transfer through the membrane, we measure the displacement of the mobile soap film. From this, we deduce the instantaneous permeability of the membrane. In contrast to the permeability of solid membranes, which inexorably decreases over time because they become clogged, an anti-clogging effect is observed with a permeability that systematically increases over time. Since the thickness of the film is constant over time, we attribute this to the possibility of adsorbing or desorbing fluorinated gas molecules on the liquid membrane. Indeed, the partial pressure of the fluorinated gas being high at the beginning of the experiment, the density of the adsorbed molecules is also high, which leads to a low permeability to air transfer. On the contrary, at the end of the experiment, the partial pressure in fluorinated gas and thus the density of the adsorbed molecules are low. This leads to a higher permeability and a less clogged membrane.

\section{Introduction}

Microbubbles are often used as ultrasound contrast agents. ${ }^{1}$ However, because of the Laplace overpressure, their lifetime in a liquid that is undersaturated with gas is limited: they empty inexorably in a few secondes. ${ }^{2}$ Several strategies are being considered to stabilize them. For example, they can be covered with a hard rigid shell that hinders their deflation. However, this strategy introduces extrinsic elements into the liquid, which can be troublesome for medical imaging applications. ${ }^{2}$ Soluble surfactants forming self-assembled monolayers at the water/air interface can also be used. These soft bubbles live longer as the Laplace overpressure is reduced due to the decrease of interfacial tension. What has been called "osmotic stabilization" can even further increase their lifetime. ${ }^{3-7}$ In that case, the microbubbles are filled with a mixture of a water-soluble gas (like air) and a water-insoluble gas (like fluorocarbon gases). The additional osmotic pressure generated by the presence of the insoluble gas then balances the Laplace pressure and stabilizes the bubbles. This strategy is also used to stabilize 
aqueous foams against coarsening. ${ }^{4-6,8-11}$ When filled with a soluble gas only, the pressure differences between adjacent bubbles of different mean curvatures induce gas transfer from high to low pressures through the thin soap films: the small bubbles thus inexorably empty into the large ones until they disappear. Filled with a mixture of soluble and insoluble gas, the bubble size distribution may find a stable equilibrium provided that the other mechanisms responsible for foam ageing, namely evaporation, coalescence and drainage, are under control. ${ }^{5,10-12}$

The characteristic time to reach this equilibrium depends on the permeability of the thin films to the soluble gas: the more permeable they are, the faster the gas transfers to reach equilibrium. In the literature, the permeability of a film to a given gas is generally measured by following the deflation of a bubble deposited on the surface of a bath when driven by Laplace overpressure. ${ }^{13-15} \mathrm{~A}$ value of the film permeability is inferred by solving a nonsteady equation for the transferred gas volume. ${ }^{15-22}$ The permeabilities measured in this way have been found to depend on different parameters: temperature, ${ }^{16}$ gas and film composition. ${ }^{18,20,21,23-27}$ However, during the deflation of the bubble, the thickness of the soap film through which gas exchange takes place is neither homogeneous nor stationary. As the film permeability is largely controlled by the thickness of the film, it is extremely difficult to compare the measured permeability with models and to extract quantitative information such as molecular-scale contributions related to the interaction between monolayers and gas molecules. To overcome this limitation, the deflation of a large hemispherical bubble above a surface has been studied ${ }^{28}$ and a stationary version of this set-up where the bubble is continuously filled with the gas under study has also been proposed. ${ }^{15,22}$ The advantage of this configuration is twofold: it allows working with a stationary film while using a gas whose composition is different from that of the atmosphere. For a mixture of methane and carbon dioxide, promising high carbon dioxide flux and separation factor have been obtained. ${ }^{22}$ However, the quantitative evaluation of this separation factor remains delicate due to the inhomogeneity in film thickness. Other configurations where the soap film is deposited in a tube are therefore used. ${ }^{17,29-32}$ In most cases, the gas flow is induced by different mechanical pressures between the two sides of the film and not driven by osmotic pressure only. Osmotic driven flows have been carried out by, ${ }^{29,30,32}$ unfortunately they have not coupled their measurements with that of the soap film thickness. In this work, we adapt this device in order to probe the effect of fluorinated gas on permeability. Monitoring the film drainage kinetics allows us to dissociate the permeability effects associated with the liquid core or surfactant layers. We show that the addition of fluorocarbon gas also has an important effect on film permeability.

\section{Experimental}

Our set-up consists of two compartments of different gas composition separated by a soap film. It is reminiscent of the one originally described in ${ }^{29}$ and later in ${ }^{17,30,33}$. The foaming solution constituting the film is either a tetradecyltrimethylammonium bromide (TTAB) solution at concentration $3 \mathrm{~g} / \mathrm{L}$ (twice the critical micellar concentration (CMC) of TTAB) or a sodium dodecylsulfate (SDS) solution at concentration 9.8 or $24.5 \mathrm{~g} / \mathrm{L}$ (4 to 10 times the CMC of SDS).

The foam film is generated manually in air in the cylindrical body of a $50 \mathrm{~mL}$ Hamilton glass syringe of length $L=6 \mathrm{~cm}$, whose inner surface is very smooth. To control the gas composition of the two compartments, we use the set-up described in Fig. 1 and proceed as follows. First, the film is brought to the top of syringe 4 by withdrawing the air above the film using syringe 3 . Syringe 4 is then placed vertically with its lower end bathed in a solution containing the foaming solution. The bottom compartment of this syringe, called compartment 1 (see Fig. 1), thus contains air at ambient pressure. Before filling the upper compartment, called compartment 2 , the film is kept at rest for a time $t_{0}$ varying from 0 to 30 minutes. During this time, 


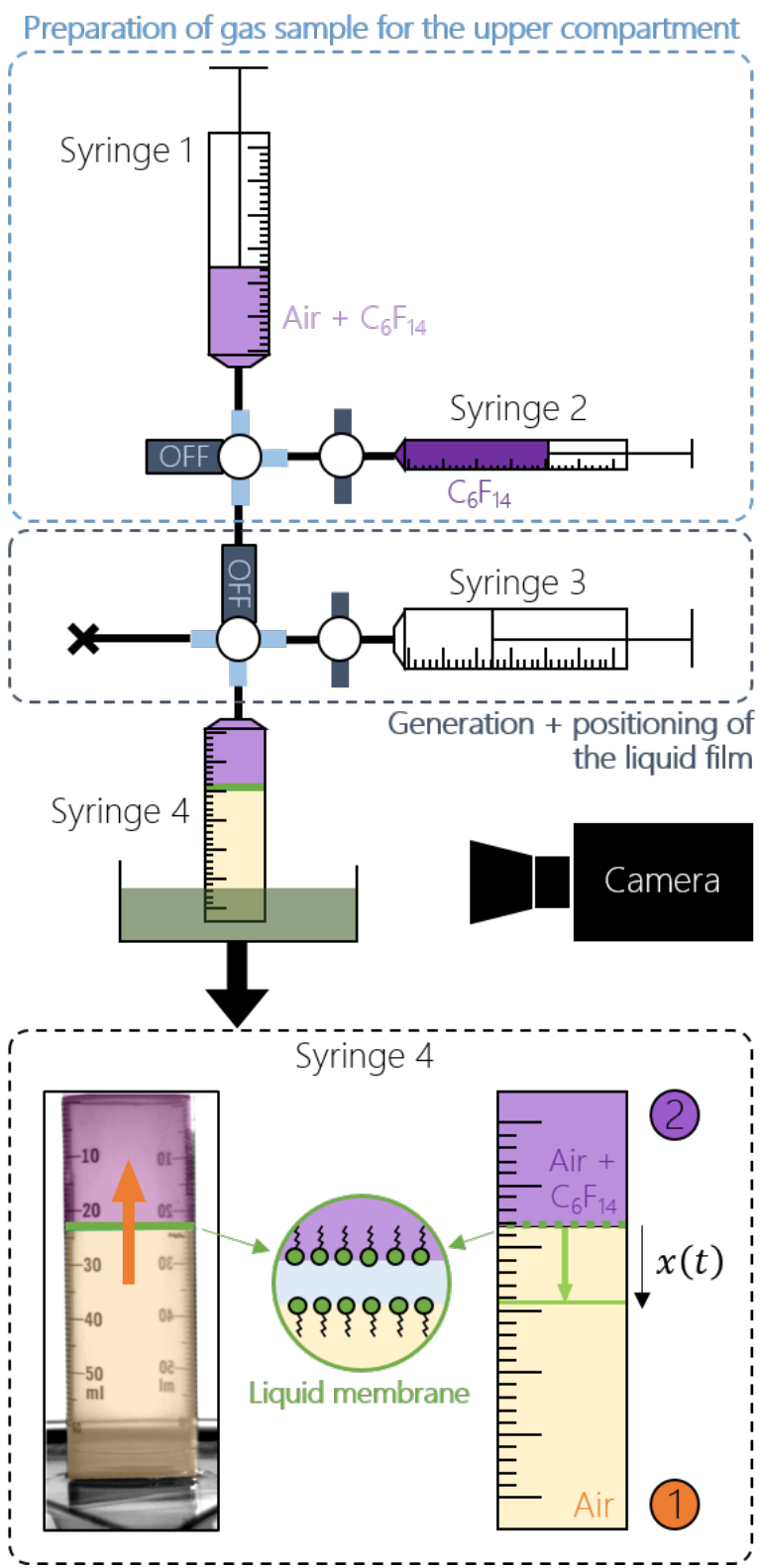

Figure 1: Top - Experimental setup used to prepare the two gas compartments and the soap film in syringe 4 (see text). Bottom - Zoom on syringe 4: The gas compartments 2 and 1 , separated by a liquid membrane composed of water and surfactants, have different compositions. As a result, the soap film moves downward. by replacing the device containing syringes $1-3$ with an optical fiber, the film thickness can be measured using an Avantes spectrometer with a wavelength between $300 \mathrm{~nm}$ and $1100 \mathrm{~nm}$. The thickness of the film typically decreases from $2000 \mathrm{~nm}$ down to less than $150 \mathrm{~nm}$ in 2 minutes. This evolution is coupled with changes in the film color: initially it is colorful and then becomes fully transparent with black circular spots appearing near the meniscus. The spectrometer range did not allow measurements below $150 \mathrm{~nm}$, but experiments in a thin film pressure balance for an horizontal film placed in a closed chamber to limit evaporation, have shown that the thickness of such films reaches a constant value of less than few tens of $\mathrm{nm}$ within less than 5 minutes. We therefore introduce $\tau$, the typical drainage time required for the film to reach this constant thickness. For the different foaming solutions we used, $\tau$ is typically equal to 5 minutes. Comparing $\tau$ and $t_{0}$ is important for our experiment: if the film is kept at rest before starting the experiment for a time $t_{0}>\tau$, we consider that the thickness of the film is constant for the duration of the experiment. Otherwise the drainage of the film is not over: The thickness will reach a constant thickness only when the age of the film equals $\tau$.

To control the gaseous composition of compartment 2, we use a procedure also described in. ${ }^{12,34}$ We fill syringe 2 with a given volume $V$ (5 to $30 \mu \mathrm{L}$ ) of $\mathrm{C}_{6} \mathrm{~F}_{14}$, which is liquid at room temperature $\left(25^{\circ} \mathrm{C}\right)$ and atmospheric pressure. This liquid volume evaporates when transferred to syringe 1 initially filled with a given volume of air. This allows us to control the initial molar fraction $\mathrm{C}_{6} \mathrm{~F}_{14}$ in syringe 1 . This gaseous contents is then rapidly transferred to syringe 3 through a system of narrow valves, which increases the flow velocity and ensures good convective homogenization of the gas mixture. After having waited for $t_{0}$, we fill syringe 4 with the content of syringe 3 . This displaces the soap film in the smooth syringe 4 until mechanical equilibrium between compartments 1 and 2 is reached. This happens in less than 5 seconds. The time $t$ is counted from the moment the film has reached this equilibrium. As 
the soap film is flat (see snapshots in Fig. 2), its mean curvature is zero and the mechanical pressure $P_{1}$ in 1 is equal to the total pressure $P_{2}$ in 2: $P_{1}=P_{2}=P_{m}$. To measure $P_{m}$, we compare the height of the liquid bath in syringe 4 and in the tank. Knowing the volume of the different dead zones of our set-up that are initially filled with air, this procedure allows us to control precisely the gaseous composition of compartment 2 whose initial partial pressure in $\mathrm{C}_{6} \mathrm{~F}_{14}$ is limited by the resolution and volume of syringe 2 . Typically $P_{\mathrm{C}_{6} \mathrm{~F}_{14}, 2}^{0}$ can be varied from 2000 to 10,000 Pa. A Thorlabs CCD camera recording at 1 frame per second follows the displacement of the soap film $x$ as a function of time $t$ (see Fig. 1 and 2). When $P_{\mathrm{C}_{6} \mathrm{~F}_{14}, 2}^{0}=0$, the soap film barely moves due to gravity (velocity smaller than $0.1 \mu \mathrm{m} / \mathrm{s}$ ). For $P_{\mathrm{C}_{6} \mathrm{~F}_{14}, 2}^{0}>2000 \mathrm{~Pa}$, it moves downward at a velocity of the order of $10 \mu \mathrm{m} / \mathrm{s}$. Images of the film displacement as well as the evolution of $x$ with time $t$ extracted from video recordings are shown for $P_{\mathrm{C}_{6} \mathrm{~F}_{14}, 2}^{0}=6800 \mathrm{~Pa}$ in Figure 2 . The film moves at nearly constant velocity until reaching the bottom of the syringe after $5000 \mathrm{~s}$ and coming into contact with the liquid bath, causing it to break.

\section{Modeling}

To quantify gas transfer through the soap film, we discuss the origin of the driving pressure on the soap film, its kinetics of displacement and then its permeability.

\section{Driving pressure}

Since $P_{1}=P_{2}$, it is not a mechanical pressure difference that causes the soap film to move, but the flow of gas between the two compartments throughout it. The solubility of $\mathrm{C}_{6} \mathrm{~F}_{14}$ in water is extremely low: its Henry coefficient $H_{\mathrm{C}_{6} \mathrm{~F}_{14}}{ }^{-}$ defined here as the ratio of the dissolved gas concentration $C_{a q}$ to its concentration in the gaseous compartment $C_{g a s}: C_{a q}=H C_{g a s}$ - is $H_{\mathrm{C}_{6} \mathrm{~F}_{14}}<2 \times 10^{-5}$ when $H_{\mathrm{O}_{2}}=3.2 \times 10^{-2}$, $H_{\mathrm{N}_{2}}=1.5 \times 10^{-2}$ for $\mathrm{O}_{2}$ or $\mathrm{N}_{2}$ in water (see ref $^{35}$ ). As a result, we consider the soap film to be impermeable to $\mathrm{C}_{6} \mathrm{~F}_{14}$. Moreover, as $H_{\mathrm{O}_{2}}$ is equivalent to $H_{\mathrm{N}_{2}}$ and their diffusion coefficients are of the same order of magnitude, we consider air as an effective gas, which is the only one that is transferred through the soap film. In compartment 1 , the partial pressure of air $P_{a i r, 1}=P_{1}=P_{m}$, while in compartment 2, $P_{a i r, 2}=P_{2}-P_{\mathrm{C}_{6} \mathrm{~F}_{14}, 2}=P_{m}-P_{\mathrm{C}_{6} \mathrm{~F}_{14}, 2}<P_{a i r, 1}$. Thus, the concentration of air in the two compartments is different, with a difference proportional to $P_{\mathrm{C}_{6} \mathrm{~F}_{14}, 2}$. This induces an air flow from compartment 1 to 2 , which in turn induces a motion of the soap film to maintain its mechanical equilibrium.

The origin of the motion can also be understood in terms of osmotic pressure as classically done for liquid solutions. ${ }^{36}$ To do so, we introduce the osmotic pressure $\Pi$ resulting from this gas transfer. On the one hand, in compartment 2 , the thermodynamical pressure is $P_{m}+\Pi$ and the air molar fraction $X_{a i r, 2}$. On the other hand, in compartment 1 , the thermodynamical pressure is $P_{m}$ and the air molar fraction $X_{a i r, 1}=$ 1. Assuming quasi-static and isothermal evolution, the chemical potential equality for air in the two compartments reads: $\mu_{a i r, 2}\left(P_{m}+\right.$ $\left.\Pi, X_{a i r, 2}\right)=\mu_{\text {air }, 1}\left(P_{m}, 1\right)$. Expanding $\mu_{a i r, 2}$ in $X_{\text {air }, 2}$ around 1 leads to: $\mu_{\text {air }, 2}\left(P_{m}+\Pi, X_{\text {air }, 2}\right)=$ $\mu_{a i r, 2}\left(P_{m}, 1\right)+R T \ln X_{a i r, 2}+R T \ln \frac{P_{m}+\Pi}{P_{m}}$ where we used in the last term the Gibbs-Duhem relation $\mathrm{d} \mu /\left.\mathrm{d} P\right|_{T}=v$ with $v$ the molar volume ( $v=R T / P$ for an ideal gas). By inserting the chemical potential equality into this expression and noting that $\ln X_{\text {air }}=\ln [1-$ $\left.X_{\mathrm{C} 6 \mathrm{~F} 14}\right] \sim-X_{\mathrm{C} 6 \mathrm{~F} 14}$ for $X_{\mathrm{C} 6 \mathrm{~F} 14}<<1$ yields: $X_{\mathrm{C} 6 \mathrm{~F} 14}=\Pi / P_{m}$. Finally, because $X_{\mathrm{C} 6 \mathrm{~F} 14}=$ $P_{\mathrm{C} 6 \mathrm{~F} 14} / P_{m}$, the latter expression leads to $\Pi=$ $P_{\mathrm{C} 6 \mathrm{~F} 14}$ which is equivalent to the expression in dilute solutions while taking into account the gas compressibility. This set-up is thus the gaseous analogue of the classical U-shaped tube equipped with a semi-permeable membrane used to demonstrate the existence of osmotic pressure in liquids: what drives the soap film is the selective gas transfer throughout the membrane rather than a mechanical pressure gradient. ${ }^{36}$

However, two differences between the gaseous and liquid experiments can be noted. First, 
the membrane in the liquid experiment is fixed, while the soap film semi-permeable membrane is mobile. As the pressure variations in the syringe are small, compressibility effects can be neglected and the soap film displacement is directly related to the transferred volume of gas. Moreover, in liquid solutions, due to the geometry of the U-shaped tube, the transferred liquid volume builds up a hydrostatic pressure, which eventually balances the osmotic pressure: a stationary regime is reached. Similarly, foams filled with a mixture of soluble and insoluble gas also find an equilibrium configuration: it is then the Laplace pressure difference between adjacent bubbles of different sizes rather than the hydrostatic pressure that balances the osmotic pressure difference. In our set-up, nothing compensates the osmotic pressure difference across the soap film membrane. As a consequence, the film never stops and slides downward until coalescing with the foaming solution of the bath as observed experimentally.

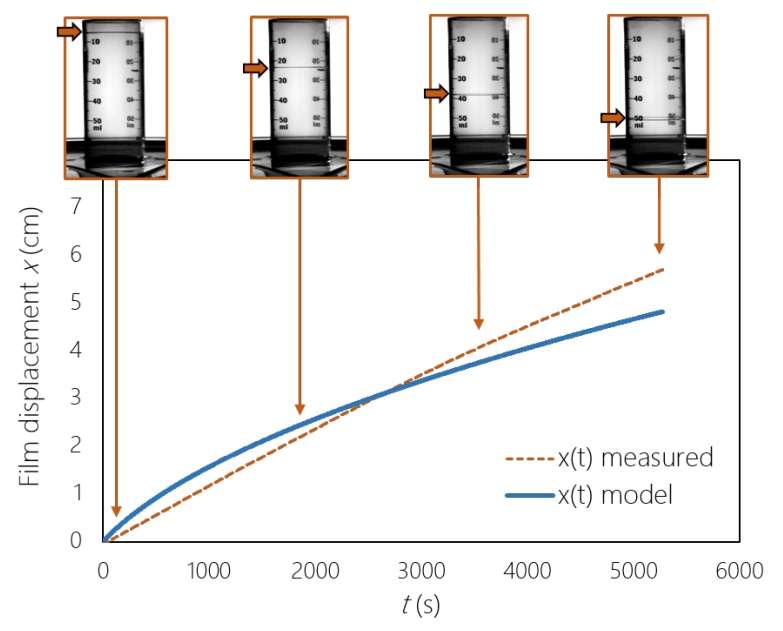

Figure 2: Soap film displacement $x$ as a function of $t$. Orange dotted line: experiment for $P_{\mathrm{C}_{6} \mathrm{~F}_{14}, 2}^{0}=6800 \mathrm{~Pa}$ and $t_{0}=10$ minutes. Thick blue line: best fit according to equation (4). The images show the displacement of the soap film in the cylindrical syringe.

\section{Kinetics}

We now focus on the kinetics of the gas transfer. Following Princen, we write a linear relation between the rate of transport and the concentration difference and define $k$ the permeability coefficient of the film to air as: ${ }^{14}$

$$
\frac{d N}{d t}=-A k \Delta C_{a i r}
$$

where $N$ is the number of moles of gas diffusing through the film of area $A$ from compartment 1 to compartment 2 , and $\Delta C_{a i r}=C_{a i r, 1}-C_{a i r, 2}$ is the difference of air concentration in the two compartments. Note that as defined, this permeability coefficient $k$ is expressed in $\mathrm{m} / \mathrm{s}$ and is therefore different from the permeability expressed in $\mathrm{m}^{2}$ conventionally used to describe fluid transport in porous media. Using ideal gas law, we write: $R T \Delta C_{\text {air }}(t)=P_{a i r, 1}(t)-$ $P_{\text {air }, 2}(t)=\Pi=P_{\mathrm{C}_{6} \mathrm{~F}_{14}, 2}$ and $R T d N / d t=$ $P A d x / d t$ where $x$ is the film displacement. Equation (1) therefore writes:

$$
\frac{d x}{d t}=k \frac{P_{\mathrm{C}_{6} \mathrm{~F}_{14}, 2}}{P_{m}}
$$

similarly to what has been proposed by Princen in the geometry of the diminishing bubble experiment. ${ }^{14}$ Using mass conservation of $\mathrm{C}_{6} \mathrm{~F}_{14}$ in compartment 2 yields $P_{\mathrm{C}_{6} \mathrm{~F}_{14}, 2}=P_{\mathrm{C}_{6} \mathrm{~F}_{14}}^{0} \frac{\xi_{0}}{\xi_{0}+x}$ where $\xi_{0}$ is the initial position of the film. Eq. (2) finally writes:

$$
\frac{d x}{d t}\left(1+\frac{x}{\xi_{0}}\right)=k \frac{P_{\mathrm{C}_{6} \mathrm{~F}_{14}}^{0}}{P_{m}} .
$$

Assuming that the permeability $k$ has a constant value for a given foaming solution/gas couple as generally proposed in literature, and since $P_{\mathrm{C}_{6} \mathrm{~F}_{14}}^{0} / P_{m}$ is a constant parameter, equation (3) can be simply solved:

$$
x(t)=\xi_{0}(\sqrt{1+\alpha t}-1),
$$

where $\alpha=2 k P_{\mathrm{C}_{6} \mathrm{~F}_{14}}^{0} / P_{m}$ is a constant. In figure 2, we compare the experimental data with equation (4). We calculate $\alpha$ as the value leading to the best fit for the model from Eq. 4 using a Scilab program, which optimizes the value for $\alpha$ using a mean square method. We obtain a poor agreement between the two curves suggesting that the constant permeability hypothesis is not valid during one experiment. As the displacement rate is nearly constant despite the 
fact that $P_{\mathrm{C}_{6} \mathrm{~F}_{14}, 2}$ decreases over time, we conclude that $k$ increases over time.

\section{Discussion}

We now recall the existing theory concerning the permeability of a thick soap film. Then, we extract the evolution of $k$ with time $t$ and compare the theory with our results. This allows us to highlight the influence of the vapour composition on the permeability. We eventually test the influence of various parameter, $P_{\mathrm{C}_{6} \mathrm{~F}_{14}}^{0}, t_{0}$ and the membrane composition.

\section{Permeability theory}

The permeability of a thick soap film can be schematically represented as a central liquid layer of thickness $e$ sandwiched by two independent monolayers of surfactant molecules. ${ }^{14,16,19,25,37-39}$ At equilibrium, the film permeability $k$ to a gas $G$ is therefore the sum of the contributions from these different layers:

$$
\frac{1}{k}=\frac{1}{H}\left(\frac{e}{D}+\frac{2}{k^{M L}}\right),
$$

where $D$ is the diffusion coefficient of the dissolved gas $G$ in the foaming solution and $H$ the Henry coefficient comparing the dissolved gas concentration $C_{a q}$ as compared to its concentration in the gaseous compartment $C_{\text {gas }}$ : $C_{a q}=H C_{g a s}$, while $k^{M L}$ is the permeability associated to the surfactant monolayers due the existence of an energy barrier at the gas-liquid interface for the transfer of the gas. It depends on the probability for a gas molecule to find a hole in the surfactant monolayer; the global permeability of a monolayer is therefore the sum of the permeability of the holeless layer and of the permeability of all holes. ${ }^{17,19}$ This framework has proven useful to quantify the evolution of the permeability of soap film to gases highly soluble in water. In particular, it has been shown that the permeability $k$ varies with $e$ as proposed in Eq. (5), with $k^{M L}$ constant for films thicker than $100 \mathrm{~nm},{ }^{33}$ i.e. for films with a liquid core thick enough to consider the two monolayers as independent. For films with a thickness between $20 \mathrm{~nm}$ and $100 \mathrm{~nm}$ (commonly called common black films), the two monolayers are not independent and this linear dependence is no longer verified: $k^{M L}$ depend on the thickness of the liquid core. ${ }^{16,18,20,25,40,41}$ In conclusion, if $e$ is constant like $H, D$ and $k^{M L}$ are also constant, then $k$ should keep a constant value, independent of time.

\section{Comparison with the data}

To understand the discrepancy between the model and the data of Fig. 2, we fit the data piecewise using Equation (3), and extract the evolution of $k$ with time.

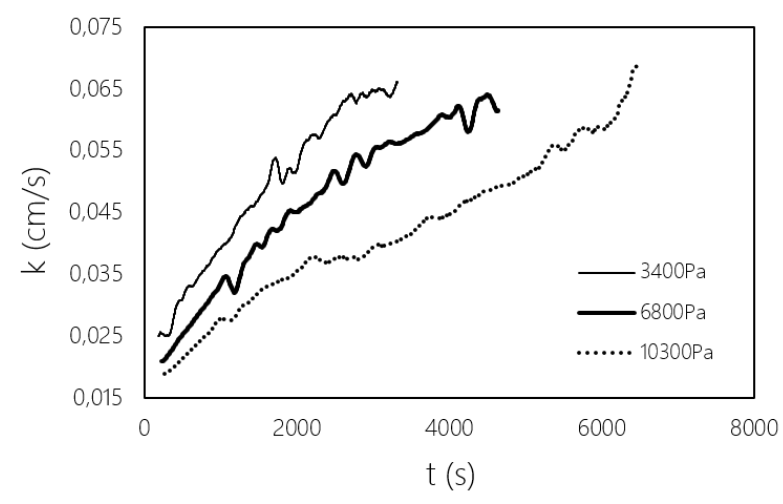

Figure 3: Evolution of the film permeability over time for different initial partial pressures $P_{\mathrm{C}_{6} \mathrm{~F}_{14}}^{0}$. The soap films are made of TTAB at a concentration $3 \mathrm{~g} / \mathrm{L}$ and $t_{0}=10$ minutes.

In Fig. 3, we show the time evolution of $k$ for $t_{0}=10 \mathrm{mn}$ and for three different $P_{\mathrm{C}_{6} \mathrm{~F}_{142}}^{0}$. For the three curves, $k$ typically increases from 0.02 $\mathrm{cm} / \mathrm{s}$ to $0.06 \mathrm{~cm} / \mathrm{s}$, throughout the two-hour long experiment. Yet, as $t_{0}>\tau$, the film thickness $e$ is constant over time and film thickness variation cannot explain the observed variations of $k$. Moreover, a closer look on the data in Fig. 3 reveals the following features. At the beginning of the films displacement i.e. for $t \sim 0$ in Fig. 3, the higher $P_{\mathrm{C}_{6} \mathrm{~F}_{14}}^{0}$, the lower $k$ is, despite the fact that these three films have exactly the same age $\left(t_{0}=10 \mathrm{~min}\right)$ and thus initially the same thickness. On the contrary, at the end of the films displacement, the permeabilities values are equivalent for the three $P_{\mathrm{C}_{6} \mathrm{~F}_{14}}^{0}$ tested, 
despite the fact that theses three films have different ages since they reach the end of the syringe at $t=3500 \mathrm{~s}, 4500 \mathrm{~s}$ and $6500 \mathrm{~s}$. This can be understood by considering that the increase of $k$ over time can be mainly attributed to the evolution of the vapour composition, i.e. the evolution of $P_{\mathrm{C}_{6} \mathrm{~F}_{14}, 2}$ over time. At the beginning of the three experiments, i.e. for $t \sim 0$ in Fig. 3, the three partial pressures of the fluorocarbon in compartment 2 are different because the $P_{\mathrm{C}_{6} \mathrm{~F}_{14}}^{0}$ values are different, whereas at the end of the experiment the fluorocarbon gas has been diluted by the transfer of air between the two compartments and the relative variations in fluorocarbon pressure between the three experiments are much smaller.

\section{Fluorocarbon pressure}

As $H$ and $D$ are not surfacic parameters and as C6F14 is insoluble, the only way to relate $k$ to $P_{\mathrm{C}_{6} \mathrm{~F}_{14}, 2}$ in Eq. 5 is through the parameter $k^{M L}$.

To explain the increase of $k^{M L}$ in time observed here while remaining within the framework of its theoretical description, one can imagine that both the concentration of surfactant in the film and the surface density of surfactants in the layer are not constant during the experiment due to water evaporation. However, given the high concentrations of surfactants used (2 to 10 times CMC) and the fact that we work in a close environment, it is unlikely that this assumption is true. The origin of the observed behaviour is more likely due to subtle interactions between the carbon chain of the apolar surfactant and the gaseous molecules of apolar $\mathrm{C}_{6} \mathrm{~F}_{14}$. Volatile oil molecules and fluorocarbon gases present in a vapour phase are known to modify the properties and behavior of various self-assembled monolayers constitued of phospholipids, surfactants, amphiphilic proteins or copolymers. ${ }^{42-45}$ Dynamic bubble profile analysis or surface pressure measurements within a Langmuir trough have shown that they can lower interfacial tensions, ${ }^{42,46,47}$ significantly accelerate the kinetics of phospholipid adsorption or modify the concentration of adsorbed surfactants at the interface, ${ }^{42,48}$ control phospholipid versus protein competi- (a)
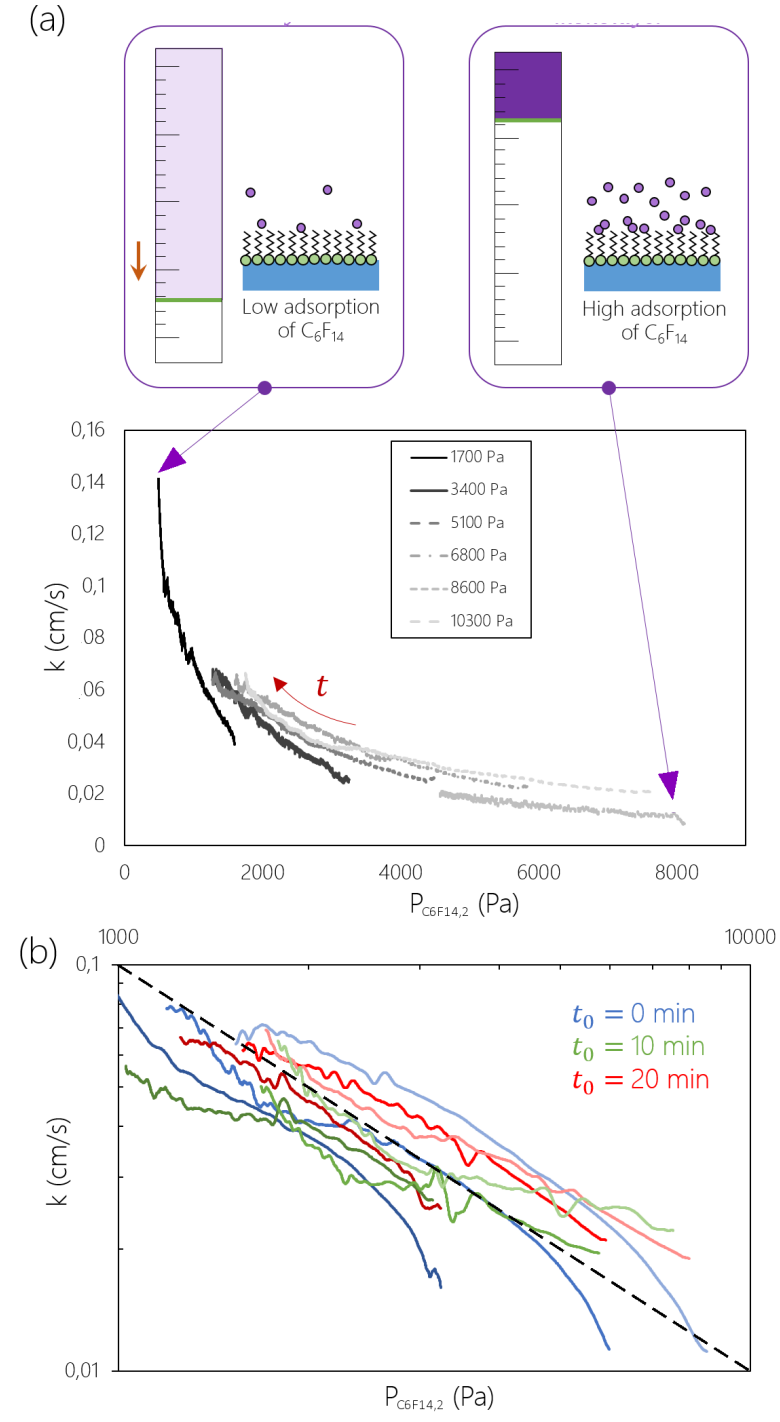

Figure 4: (a) Bottom: Evolution of $k$ as a function of $P_{\mathrm{C}_{6} \mathrm{~F}_{14}, 2}$ for various $P_{\mathrm{C}_{6} \mathrm{~F}_{14}}^{0}$ and $t_{0}=10$ min. Top: Thumbnails showing the additional permeability induced by the adsorption of fluorinated molecules on the membrane, the concentration of which increases with $P_{\mathrm{C}_{6} \mathrm{~F}_{14}, 2}$. (b) Log-log plot of $k$ as a function of $P_{\mathrm{C}_{6} \mathrm{~F}_{14}, 2}$ for various $P_{\mathrm{C}_{6} \mathrm{~F}_{14}}^{0}$ and various $t_{0}$ : The different values of $t_{0}$ are indicated by different colors: $0 \mathrm{~min}$ (blue), $10 \mathrm{~min}$ (green) and $20 \mathrm{~min}$ (red). For each $t_{0}$, three different $P_{\mathrm{C}_{6} \mathrm{~F}_{14}}^{0}$ are tested 3400 $\mathrm{Pa}, 6800 \mathrm{~Pa}$ and $10300 \mathrm{~Pa}$ (the lighter the color, the higher $\left.P_{\mathrm{C}_{6} \mathrm{~F}_{14}}^{0}\right)$. The black dotted line is a guide for the eyes showing the empirical scaling $k \sim P_{\mathrm{C}_{6} \mathrm{~F}_{14}, 2}^{-1}$.

tive adsorption ${ }^{49}$ or alter the vibrating properties of microbubbles, ${ }^{50}$ highlighting the transformation of the bubble shells due to gas ad- 
sorption on the inner interface. Fluorocarbon molecules usually do not exhibit a polar head nor amphiphilic properties, yet these observations have clearly established that they act as co-surfactants and adsorb at the air/water interface. ${ }^{45,51}$

In our experiment, the presence of an additional adsorbed layer would indeed decrease the permeability of the system since it would add an additional barrier that would resist the passage of air molecules, similar to that created by surfactant layers. Contrary to most of previous studies, the partial pressure of $\mathrm{C}_{6} \mathrm{~F}_{14}$ is not constant in our experiments. Linking the additional membrane resistance due to adsorbed fluorinated molecules to their partial pressure is a difficult task, which likely requires neutron or X-ray reflectometry studies well beyond the scope of this work. ${ }^{51,52}$ However, for the sake of simplicity, it can be assumed that adsorption is reversible and rapid in view of the characteristic gas transfer time (of the order of $L / k \sim 10$ s). Under this assumption, the concentration of adsorbed molecules on the membrane as well as the permeability would only depend on the fluorinated gas pressure in compartment $2, P_{\mathrm{C}_{6} \mathrm{~F}_{14}, 2}$. All the permeability data should then collapse on a master curve when plotted as a function of $P_{\mathrm{C}_{6} \mathrm{~F}_{14}, 2}$ rather than as a function of time. This is indeed what we observe for TTAB, as shown in Fig. 4a where data corresponding to different initial partial pressures in $\mathrm{C}_{6} \mathrm{~F}_{14}$ are reported for $t_{0}=10 \mathrm{~min}$. They all overlap reasonably well, showing that the variation of permeability with time depends mainly on the partial pressure of $\mathrm{C}_{6} \mathrm{~F}_{14}$ in compartment 2 . The increase of $k$ can then be explained as follows: over time, more and more air molecules pass through the membrane to balance the partial pressure of $\mathrm{C}_{6} \mathrm{~F}_{14}$ in compartment 2. As a result, $P_{\mathrm{C}_{6} \mathrm{~F}_{14}, 2}$ decreases and the density of adsorbed molecules on the membrane also decreases. The additional resistance to air flow is less important, the membrane becomes more permeable. Overall, the filter is less clogged at the end of the experiment than at the beginning!

\section{Influence of $t_{0}$}

To check whether $t_{0}$ influences the collapse observed in Fig. 4a), we plot in Figure $4 \mathrm{~b}$ ), $k$ as a function of $P_{\mathrm{C}_{6} \mathrm{~F}_{14}, 2}$ for three sets of data concerning three different values of $t_{0}\left(t_{0}=0 \mathrm{~min}\right.$ in blue, $t_{0}=10 \mathrm{~min}$ in green and $t_{0}=20 \mathrm{~min}$ in red). For each color, the different curves correspond to different initial values of $P_{\mathrm{C}_{6} \mathrm{~F}_{14}}^{0}=3400$ $\mathrm{Pa}, 6800 \mathrm{~Pa}$ or $10300 \mathrm{~Pa}$. We can see that in $\log / \log$ scale, the two data sets corresponding to $t_{0}>\tau=5 \mathrm{~min}$ (represented in green and red) all follow an empirical power law curve with a slope of the order of -1 for the whole range of $P_{\mathrm{C}_{6} \mathrm{~F}_{14}, 2}$ and whatever the value of $P_{\mathrm{C}_{6} \mathrm{~F}_{14}}^{0}$ (this empirical law $k \sim P_{\mathrm{C}_{6} \mathrm{~F}_{14}, 2}{ }^{-1}$ being highlighted by a black dotted line in Fig. 4b). Yet, the data set corresponding to $t_{0}<\tau=5 \mathrm{~min}$ (represented in blue) only follows this $k \sim P_{\mathrm{C}_{6} \mathrm{~F}_{14}, 2}{ }^{-1}$ trend for the smallest values of $P_{\mathrm{C}_{6} \mathrm{~F}_{14}, 2}$. For larger values, the permeability is systematically lower - for instance when comparing the three curves corresponding to $P_{\mathrm{C}_{6} \mathrm{~F}_{14}}^{0}=6800 \mathrm{~Pa}$ for $P_{\mathrm{C}_{6} \mathrm{~F}_{14}, 2} \sim 5000 \mathrm{~Pa}$, the permeability value for $t_{0}=0 \mathrm{~min}$ is slightly above $0.01 \mathrm{~cm} / \mathrm{s}$, while for $t_{0}=10 \mathrm{~min}$ and $20 \mathrm{~min}$ it is around 0.02 $\mathrm{cm} / \mathrm{s}$. The $\log / \log$ scale therefore logically reveals that for the larger values of $P_{\mathrm{C}_{6} \mathrm{~F}_{14}, 2}$ the slope of the experimental data is systematically smaller than -1 . This can be explained by recalling that i) $P_{\mathrm{c}_{6} \mathrm{~F}_{14}, 2}$ is a decreasing function of time $t$ (short times correspond to large values of $P_{\mathrm{c}_{6} \mathrm{~F}_{14}, 2}$ and long times to small values) ii) for $t_{0}=0$, the film has not fully drained and is thus thicker than at equilibrium. Thus, at short times $t$ or for high $P_{\mathrm{C}_{6} \mathrm{~F}_{14}, 2}$ values, this additional thickness leads to an additional resistance to gas transfer and thus to a lower permeability. On the contrary, at long times or low $P_{\mathrm{C}_{6} \mathrm{~F}_{14}, 2}$ values, the film has reached its equilibrium thickness and the permeability curves for $t_{0}=0$ min catch up with those observed for $t_{0}>\tau$.

\section{Membrane composition}

As previously discussed, the influence of fluorocarbon gases on surface tension and on the organization of self-assembled layers was tested 


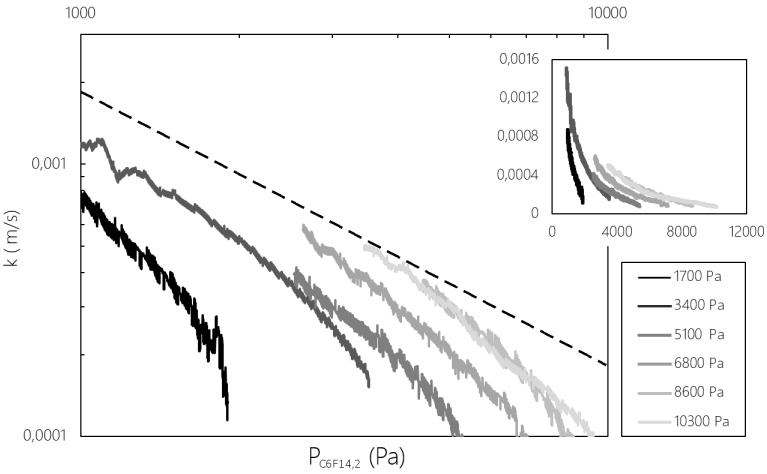

Figure 5: Log-log plot of SDS film permeability as a function of $P_{\mathrm{C}_{6} \mathrm{~F}_{14}, 2}$ for $t_{0}=10 \mathrm{~min}$ and different $P_{\mathrm{C}_{6} \mathrm{~F}_{14}}^{0}$. The black dotted line is a guide for the eyes showing the empirical scaling $k \sim P_{\mathrm{C}_{6} \mathrm{~F}_{14}}^{-1}$. Insert: lin-lin representation of the same data. Note that for each curve, the maximal $P_{\mathrm{C}_{6} \mathrm{~F}_{14}, 2}$ is always slightly smaller than the indicated $P_{\mathrm{C}_{6} \mathrm{~F}_{14}}^{0}$ due to data averaging.

on a wide variety of molecules: phospholipids, surfactants, amphiphilic proteins or copolymers, ${ }^{51} .{ }^{45}$ The extent to which the reduction in permeability of TTAB-stabilized membranes in the presence of $\mathrm{C}_{6} \mathrm{~F}_{14}$ is observed for other surfactants is an interesting question. As TTAB is a cationic surfactant, we also perform soap films stabilized with SDS, which is an ionic surfactant.

Similarly to TTAB-stabilized membranes, the permeability of SDS-stabilized membranes decrease with $P_{\mathrm{C}_{6} \mathrm{~F}_{14}, 2}$ as can be seen in the inset of Fig. 5 for $t_{0}=10 \mathrm{~min}$, thus confirming the observed effect for an anionic surfactant. However, there is no collapse of the curves for SDS-stabilized membrane, unlike for TTABstabilized membranes. Moreover, the same data plotted in $\log / \log$ scale reveal the same trend as that of films stabilized by TTAB for $t_{0}=0$ min: The decrease in permeability with $P_{\mathrm{C}_{6} \mathrm{~F}_{14}, 2}$ is much greater for high values of $P_{\mathrm{C}_{6} \mathrm{~F}_{14}, 2}$ (i.e. for short time) than for low values (i.e. for long time) (see Fig. 5). This may suggest than the thickness of SDS stabilized film is not constant. This is surprising since the data in Fig. 5 correspond to $t_{0}>\tau$. However, we must emphasize that the drainage kinetics of the films is measured in the absence of fluorocarbon environments. The presence of $\mathrm{C}_{6} \mathrm{~F}_{14}$ could slow down the drainage kinetics of SDS-stabilized films. This specific behavior of SDS and $\mathrm{C}_{6} \mathrm{~F}_{14}$ would find its origin in the hydrolysis of SDS into dodecanol: The slow kinetics of this hydrolysis of the order of a few hours - confers on SDS a surface rheological behavior more complex than that of TTAB. Note that a commercial foaming product - Fairy Liquid - also exhibits a decrease of $k$ with $P_{\mathrm{C}_{6} \mathrm{~F}_{14}, 2}$ but again with differences in the shape of the curves. This suggests that the molecular scale interaction between molecules of surfactant and fluorinated gases are crucial.

The universality of the decrease in membrane permeability with increasing $\mathrm{C}_{6} \mathrm{~F}_{14}$ partial pressure effect whatever the surfactant used, is indirectly confirmed by experiments reporting the abnormally long lifetime of microbubbles in water. ${ }^{47,53}$ For instance, bubbles of a few microns in radius stabilized with dimyristoylphosphatidylcholine phospholipids DMPC were observed for more than one hour, whereas the permeabilities measured without fluorinated gases predicted a lifetime of the order of one minute. This effect is also observed for dipalmitoylphosphatidylcholine (DPPC) phospholipids. ${ }^{47,53}$

Whether this observation is valid whatever the surfactant used is also important for" osmotic stabilization" of bubbles and liquid foams against coarsening (already presented in the introduction). To limit the gas transfer between bubbles of different internal capillary pressure, they are often blown with a mixture of soluble and insoluble gas such as air and $\mathrm{C}_{6} \mathrm{~F}_{14}$. The additional osmotic pressure generated by the difference in concentration of fluorocarbon gases balance the capillary pressure differences, thereby arresting coarsening. However, our work highlights that this stabilizing mechanism can also find its origins in the decreased permeability subsequent to fluorocarbons adsorption on the liquid films. This adsorption strongly attenuates gas transfer kinetics thus also limiting coarsening. Adding $\mathrm{C}_{6} \mathrm{~F}_{14}$ would therefore have the effect of both modifying the equilibrium configuration of the system while also considerably slowing down its kinetics of evolution. 


\section{Conclusions}

Using a set-up equivalent to the one used to measure the osmotic pressure of a liquid solution, we show that the permeability of a soap film membrane to the soluble specie (here air) depends on the partial pressure of the insoluble specie (here $\mathrm{C}_{6} \mathrm{~F}_{14}$ ). This indicates that the measurement of a macroscopic parameter such as membrane permeability can reveal subtle interactions at the molecular level between the membrane and its atmosphere. Understanding the fine nature of these molecular scale interactions remains an open and challenging scientific question. Moreover, as this interaction controls the intensity of the change in permeability, this could be used in chemical engineering for optimizing gas and nanoparticle separation processes. These are generally performed via complex and expensive porous membranes facing clogging issues. Breaking these current technological limitations relies on decreasing the costs and simplifying the existing protocols. A good alternative thus lies in specific liquid materials such as thin liquid films.

Acknowledgement The authors thank C. Picard, T. Metivet, T. Combriat, C. Latargez, R. Bollache, T. Orlova, S. Spagnoli for helpful discussions. This work has been partially supported by the LabEx Tec 21 (Investissements d'Avenir - grant agreement $\mathrm{n}^{o}$ ANR-11-LABX-0030) and the French National Research Agency in the framework of the "Investissement d'avenir" program (ANR-15IDEX-02). Wiebke Drenckhan acknowledges exchanges with Marie-Pierre Krafft and funding from the Institut Charnot MICA (Project ThiFilAn).

\section{References}

(1) Qin, S.; Caskey, C. F.; Ferrara, K. W. Ultrasound contrast microbubbles in imaging and therapy: physical principles and engineering. Phys. Med. Biology 2009, 54, R27-R57.

(2) Schutt, E.; Klein, D.; Mattrey, R.;
Riess, J. Injectable microbubbles as contrast agents for diagnostic ultrasound imaging: The key role of perfluorochemicals. Angew. Chem.-Int. Ed. 2003, 42, 3218-3235.

(3) Reiss, H.; Koper, G. The Kelvin relation Stability, fluctuation, and factors involved in measurement. J. Phys. Chem. 1995, 99, 7837-7844.

(4) Webster, A.; Cates, M. Stabilization of emulsions by trapped species. Langmuir 1998, 14, 2068-2079.

(5) Webster, A.; Cates, M. Osmotic stabilization of concentrated emulsions and foams. Langmuir 2001, 17, 595-608.

(6) Gandolfo, F.; Rosano, H. Interbubble gas diffusion and the stability of foams. $J$. Colloid Interface Sci. 1997, 194, 31-36.

(7) Kabalnov, A.; Klein, D.; Pelura, T.; Schutt, E.; Weers, J. Dissolution of multicomponent microbubbles in the bloodstream: 1. Theory. Ultrasound Med. Biol. 1998, 24, 739-749.

(8) Weaire, D.; Pageron, V. Frustated froth Evolution of foam inhibited by an insoluble gaseous component. Phil. Mag. 1990, 62, 417-421.

(9) Saint-Jalmes, A. Physical chemistry in foam drainage and coarsening. Soft Matter 2006, 2, 836-849.

(10) Cantat, I.; Cohen-Addad, S.; Elias, F.; Graner, F.; Höhler, R.; Pitois, O.; Rouyer, F.; Saint-Jalmes, A. Foams: Structure and Dynamics; Oxford University Press, 2013.

(11) Stevenson, P. Foam Engineering: Fundamentals and Applications; J. Wiley, 2012.

(12) Bey, H.; Wintzenrieth, F.; Ronsin, O.; Höhler, R.; Cohen-Addad, S. Stabilization of foams by the combined effects of an insoluble gas species and gelation. Soft Matter 2017, 13, 6816-6830. 
(13) Brown, A.; Thuman, W. C.; McBain, J. Transfer of air through adsorbed surface films as a factor in foam stability. J. Colloid Sci. 1953, 8, 508-519.

(14) Princen, H.; Mason, S. G. The permeability of soap films to gases I. J. Colloid Interface Sci. 1965, 20, 353-375.

(15) Nedyalkov, M.; Krustev, R.; Kashchiev, D.; Platikanov, D.; Exerowa, D. Permeability of Newtonian black foam films to gas. Colloid Polymer Sci. 1988, 266, 291-296.

(16) Krustev, R.; Platikanov, D.; Nedyalkov, M. Permeability of common black foam films to gas. Part 2. Colloids Surf. A 1997, 123-124, 383-390.

(17) Nguyen, P. Q.; Zitha, P.; Currie, P. Effect of foam films on gas diffusion. J. Colloid Interface Sci. 2003, 125, 1128-1129.

(18) Muruganathan, R.; Müller, H. J.; Möhwald, H.; Krastev, R. Effect of headgroup size on permeability of newton black films. Langmuir 2005, 21, 12222-12228.

(19) Farajzadeh, R.; Krastev, R.; Zitha, P. Foam film permeability: Theory and experiment. Adv. Colloid Interface Sci. 2008, 137, 27-44.

(20) Farajzadeh, R.; Muruganathan, R.; Rossen, W. R.; Krastev, R. Effect of gas type on foam film permeability and its implications for foam flow in porous media. Adv. Colloid Interface Sci. 2011, $168,71-78$.

(21) Ramanathan, M.; Müller, H.; Möhwald, H.; Krastev, R. Foam Films as Thin Liquid Gas Separation Membranes. ACS Appl. Mater. Interfaces 2011, 3, 633-637.

(22) Reznickova, J.; Petrychkovych, R.; Vejrazka, J.; Setnickova, K.; Uchytil, P. Gas separation ability of the liquid bubble film. Sep. Purif. Technol. 2016, 166, 26-33.
(23) Saulnier, L.; Drenckhan, W.; Larré, P.; Anglade, C.; Langevin, D.; Janiaud, E.; Rio, E. In situ measurement of the permeability of foam films using quasi-twodimensional foams. Colloids Surf. A 2015, 473, 32-39.

(24) Nedyalkov, M.; Krustev, R.; Stankova, A.; Platikanov, D. Mechanism of permeation of gas through Newton black films at different temperatures. Langmuir 1992, 8, 3142-3144.

(25) Krustev, R.; Platikanov, D.; Nedyalkov, M. Permeability of common black foam films to gas. Part 1. Colloids Surf. A 1993, 79, 129-136.

(26) Krustev, R.; Platikanov, D.; Stankova, A.; Nedyalkov, M. Permeation of gas through Newton Black Films at different chain length of the surfactant. J. Disper. Sci. Technol. 1997, 18, 789-800.

(27) Briceno-Ahumada, Z.; Langevin, D. On the influence of surfactant on the coarsening of aqueous foams. Adv. Colloid Interface Sci. 2017, 244, 124-131.

(28) Krustev, R.; Muller, H. An apparatus for the measurement of the gas permeability of foam films. Rev. Sci. Instr. 2002, 73, 398-403.

(29) Cook, R. L.; Tock, R. W. Aqueous Membranes for the Separation of Gaseous Mixtures. Sep. Sci. 1974, 9, 185-193.

(30) Haas, F. W.; Tock, R. W. Permeation of Permanent Gases through Liquid Membranes. Sep. Sci. 1975, 10, 723-729.

(31) Uchytil, P.; Setnickova, K.; Tseng, H.-H.; Sima, V.; Petrickovic, R. Description of the gas transport through dynamic liquid membrane. Sep. Purif. Technol. 2017, $184,152-157$.

(32) Sujatha, K.; Das, T.; Kumar, R.; Gandhi, K. Permeation of gases through liquid films. Chem. Eng. Sci. 1988, 43, 1261-1268. 
(33) Hadji, C.; Dollet, B.; Coasne, B.; Lorenceau, E. Specific permability of foam film. In preparation 2020,

(34) Yip Cheung Sang, Y.; Lorenceau, E.; Wahl, S.; Stoffel, M.; Angelescu, D. E.; Hoehler, R. A microfluidic technique for generating monodisperse submicron-sized drops. $R S C A d v$. 2013, 3, 2330-2335.

(35) Wilhem, E.; Battino, R.; Wilcock, R. Lowpressure solubility of gases in liquid water. Chem. Rev. 1977, 7r, 219-262.

(36) van 't Hoff, J. H. The role of osmotic pressure in the analogy between solutions and gases. J. Membr. Sci. 1995, 100, 39-44.

(37) Langmuir, I.; Langmuir, D. B. The Effect of Monomolecular Films on the Evaporation of Ether Solutions. J. Phys. Chem. 1927, 31, 1719-1731.

(38) Langmuir, I.; Schaefer, V. J. Rates of evaporation of water through compressed monolayers on water. J. Franklin Institute 1943, 235, 119-162.

(39) Princen, H.; Overbeek, J. T. G.; Mason, S. G. The permeability of soap films to gases II. J. Colloid Interface Sci. 1967, 24, 125-130.

(40) Farajzadeh, R.; Krastev, R.; Zitha, P. L. J. Gas Permeability of Foam Films Stabilized by an alpha-Olefin Sulfonate Surfactant. Langmuir 2009, 25, 2881-2886.

(41) Farajzadeh, R.; Krastev, R.; Zitha, P. L. J. Foam films stabilized with alpha olefin sulfonate (AOS). Colloids Surf. A 2008, 324, $35-40$.

(42) Mucic, N.; Moradi, N.; Javadi, A.; Aksenenko, E. V.; Fainerman, V. B.; Miller, R. Mixed adsorption layers at the aqueous C(n)TAB solution/hexane vapour interface. Colloids Surf. A 2014, 442, 50-55.

(43) Javadi, A.; Moradi, N.; Fainerman, V. B.; Moehwald, H.; Miller, R. Alkane vapor and surfactants co-adsorption on aqueous solution interfaces. Colloids Surf. A 2011, 391, 19-24.

(44) Arangalage, M.; Li, X.; Lequeux, F.; Talini, L. Dual Marangoni effects and detection of traces of surfactants. Soft Matter 2018, 14, 3378-3386.

(45) Counil, C.; Krafft, M. Organofluorine Physical Chemistry in Frontiers Of Organofluorine Chemistry; World scientific, 2019.

(46) Nguyen, P.; TTD, T.; Waton, G.; Vandamme, T.; Krafft, M.-P. A Nonpolar, Nonamphiphilic Molecule Can Accelerate Adsorption of Phospholipids and Lower Their Surface Tension at the Air/Water Interface. ChemPhysChem 2011, 12, 2646-2652.

(47) Shi, D.; Counil, C.; Krafft, M.-P. Fluorocarbon exposure mode markedly affects phospholipid monolayer behavior at the gas/liquid interface: Impact on size and stability of microbubbles. Langmuir 2018, 32, 12461-12467.

(48) Rossi, S.; Waton, G.; Krafft, M. Small phospholipid-coated gas bubbles can last longer than larger ones. ChemPhysChem 2008, 9, 1982-1985.

(49) Nguyen, P.; Veschgini, M.; Tanaka, M.; Waton, G.; Vandammec, T.; Krafft, M. Counteracting the inhibitory effect of proteins towards lung surfactant substitutes: a fluorocarbon gas helps displace albumin at the air/water interface. Chem. Commun. 2014, 50, 11576-11579.

(50) Ando, Y.; Tabata, H.; Cagna, A.; Koyama, D.; Krafft, M. P. Microbubbles with a self-assembled poloxamer shell and a fluorocarbon inner gas. Langmuir 2016, 32, 12461-12467.

(51) Counil, C. Effet d'un fluorocarbure gazeux sur l'adsorption et la stabilité à l'interface air/eau de monocouches de phospholipides, de tensioactifs et de biomarqueurs. 
Ph.D. thesis, Université de Strasbourg, 2019.

(52) Mielke, S.; Abuillan, W.; Veschgini, M.; Liu, X.; Konovalov, O.; Krafft, M. P.; Tanaka, M. Influence of PerfluorohexaneEnriched Atmosphere on Viscoelasticity and Structural Order of Self-Assembled Semifluorinated Alkanes at the Air-Water Interface. ChemPhysChem 2019, 20, 1698-1705.

(53) Szijjarto, C.; Rossi, S.; Waton, G.; Krafft, M. P. Effects of Perfluorocarbon Gases on the Size and Stability Characteristics of Phospholipid-Coated Microbubbles: Osmotic Effect versus Interfacial Film Stabilization. Langmuir 2012, 28, 1182-1189. 